\title{
On the generalized orthogonal stability of mixed type additive-cubic functional equations in modular spaces
}

\author{
Iz-iddine El-Fassi*1 ${ }^{* 1}$ Samir Kabbaj ${ }^{2}$ \\ 1,2 Department of Mathematics, Faculty of Sciences, University of Ibn Tofail, Kenitra, Morocco. \\ * Corresponding author. \\ E-mail: ${ }^{1}$ izidd-math@hotmail.fr, ${ }^{2}$ samkabbaj@yahoo.fr
}

\begin{abstract}
In this paper, we establish the Hyers-Ulam-Rassias stability of the mixed type additive-cubic functional equation

$$
f(2 x+y)+f(2 x-y)-f(4 x)=2[f(x+y)+f(x-y)]-8 f(2 x)+10 f(x)-2 f(-x),
$$

with $x \perp y$, where $\perp$ is the orthogonality in the sense of Rätz in modular spaces.
\end{abstract}

2010 Mathematics Subject Classification. 39B52. 39B55, 39B82, 47H09

Keywords. Hyers-Ulam-Rassias stability, Orthogonality, Orthogonally additive-cubic equation, Modular space.

\section{Introduction}

The study of stability problems for functional equations is related to a question of Ulam [21] in 1940, concerning the stability of group homomorphisms and affirmatively answered for Banach spaces by Hyers [10]. In 1950, a generalized version of Hyers' theorem for approximate additive mapping was given by Aoki [2]. In 1978, Rassias [17] provided a generalization of Hyers Theorem which allows the Cauchy difference to be unbounded. This stability phenomenon is called the Hyers-Ulam-Rassias stability.

Stability problems for some functional equations have been extensively investigated by several authors, and in particular one of the most important functional equation in this topic is

$$
f(x+y)+f(x-y)=2 f(x)+2 f(y),
$$

which is studied by Adam [1], P. Găvruta [7], M. Eshaghi [6], and A. Najati [13].

Recently, Gh. Sadeghi [19] proved the Hyers-Ulam stability of the generalized Jensen functional equation $f(r x+s y)=r g(x)+s h(x)$ in modular spaces, using the fixed point method, also Iz. ELFassi and S. Kabbaj in [5] investigated the Hyers-Ulam-Rassias stability of (1.1) in modular spaces. The theory of modulars on linear spaces and the corresponding theory of modular linear spaces were founded by H. Nakano [14]. In the present time the theory of modulars and modular spaces is extensively applied, in particular, in the study of various Orlicz spaces [15] and interpolation theory [12]. The importance for applications consists in the richness of the structure of modular spaces, that-besides being Banach spaces (or $F$-spaces in more general setting)- are equipped with modular equivalent of norm or metric notions. Numerous papers on the stability of some functional equations have been published by different authors, we refer, for example, to [3], [4], [11] and [20]. 
There are several orthogonality notions on a real normed spaces as Birkhoff-James, Carlsson, Singer, Roberts, Pythagorean, isosceles and Diminnie. Let us recall the orthogonality space in the sense of Rätz; cf. [18].

Suppose $E$ is a real vector space with $\operatorname{dim} E \geq 2$ and $\perp$ is a binary relation on $E$ with the following properties:

(O1) totality of $\perp$ for zero: $x \perp 0,0 \perp x$ for all $x \in E$;

(O2) independence: if $x, y \in E-\{0\}, x \perp y$, then, $x, y$ are linearly independent;

(O3) homogeneity: if $x, y \in E, x \perp y$, then $\alpha x \perp \beta y$ for all $\alpha, \beta \in \mathbb{R}$;

(O4) the Thalesian property: if $P$ is a 2-dimensional subspace of $E, x \in P$ and $\lambda \in \mathbb{R}^{+}$, then there exists $y_{0} \in P$ such that $x \perp y_{0}$ and $x+y_{0} \perp \lambda x-y_{0}$.

The pair $(E, \perp)$ is called an orthogonality space. By an orthogonality normed space, we mean an orthogonality space having a normed structure. Some interesting examples of orthogonality spaces are:

(i) The trivial orthogonality on a vector space $E$ defined by (O1), and for nonzero elements $x, y \in E, x \perp y$ if and only if $x, y$ are linearly independent.

(ii) The ordinary orthogonality on an inner product space $(E,\langle\rangle$.$) given by x \perp y$ if and only if $\langle x, y\rangle=0$.

(iii) The Birkhoff-James orthogonality on a normed space $(E,\|\|$.$) defined by x \perp y$ if and only if $\|x\| \leq\|x+\lambda y\|$ for all $\lambda \in \mathbb{R}$.

The relation $\perp$ is called symmetric if $x \perp y$ implies that $y \perp x$ for all $x, y \in E$. Clearly examples (i) and (ii) are symmetric but example (iii) is not. However, it is remarkable to note, that a real normed space of dimension greater than or equal to 3 is an inner product space if and only if the Birkhoff-James orthogonality is symmetric.

The Orthogonal Cauchy functional equation

$$
f(x+y)=f(x)+f(y) \quad(x, y \in E, x \perp y)
$$

in which $\perp$ is an abstract orthogonally was first investigated by S. Gudder and D. Strawther [9]. R. Ger and J. Sikorska discussed the orthogonal stability of the equation (1.2) in [8]. S. Ostadbashi and J. Kazemzadeh [16] investigated the problem of the Orthogonal stability of the mixed additive-cubic functional equation

$$
f(2 x+y)+f(2 x-y)-f(4 x)=2[f(x+y)+f(x-y)]-8 f(2 x)+10 f(x)-2 f(-x)(x \perp y),
$$

in Banach space.

In the present paper, we establish the Hyers-Ulam-Rassias stability of orthogonally mixed additive-cubic functional equation (1.3) in modular spaces. Therefore, we generalized the main results of $[16]$.

\section{Preliminary}

In this section, we give the definitions that are important in the following. 
Definition 2.1. Let $X$ be an arbitrary vector space.

(a) A functional $\rho: X \rightarrow[0, \infty]$ is called a modular if for arbitrary $x, y \in X$,

(i) $\rho(x)=0$ if and only if $x=0$,

(ii) $\rho(\alpha x)=\rho(x)$ for every scalar $\alpha$ with $|\alpha|=1$,

(iii) $\rho(\alpha x+\beta y) \leq \rho(x)+\rho(y)$ if and only if $\alpha+\beta=1$ and $\alpha, \beta \geq 0$,

(b) if (iii) is replaced by

(iii)' $\rho(\alpha x+\beta y) \leq \alpha \rho(x)+\beta \rho(y)$ if and only if $\alpha+\beta=1$ and $\alpha, \beta \geq 0$, then we say that $\rho$ is a convex modular.

A modular $\rho$ defines a corresponding modular space, i.e., the vector space $X_{\rho}$ given by

$$
X_{\rho}=\{x \in X: \rho(\lambda x) \rightarrow 0 \text { as } \lambda \rightarrow 0\} .
$$

Let $\rho$ be a convex modular, the modular space $X_{\rho}$ can be equipped with a norm called the Luxemburg norm, defined by

$$
\|x\|_{\rho}=\inf \left\{\lambda>0: \rho\left(\frac{x}{\lambda}\right) \leq 1\right\} .
$$

A function modular is said to satisfy the $\Delta_{2}$-condition if there exists $\kappa>0$ such that $\rho(2 x) \leq$ $\kappa \rho(x)$ for all $x \in X_{\rho}$.

Definition 2.2. Let $\left\{x_{n}\right\}$ and $x$ be in $X_{\rho}$. Then

(i) we say $\left\{x_{n}\right\}$ is $\rho$-convergent to $x$ and write $x_{n} \stackrel{\rho}{\rightarrow} x$ if and only if $\rho\left(x_{n}-x\right) \rightarrow 0$ as $n \rightarrow \infty$,

(ii) the sequence $\left\{x_{n}\right\}$, with $x_{n} \in X_{\rho}$, is called $\rho$-Cauchy if $\rho\left(x_{n}-x_{m}\right) \rightarrow 0$ as $m, n \rightarrow \infty$,

(iii) a subset $S$ of $X_{\rho}$ is called $\rho$-complete if and only if any $\rho$-Cauchy sequence is $\rho$-convergent to an element of $S$.

The modular $\rho$ has the Fatou property if and only if $\rho(x) \leq \lim _{n \rightarrow \infty} \inf \rho\left(x_{n}\right)$ whenever the sequence $\left\{x_{n}\right\}$ is $\rho$-convergent to $x$.

Remark 2.3. If $x \in X_{\rho}$ then $\rho(a x)$ is a nondecreasing function of $a \geq 0$. Suppose that $0<a<b$, then property (iii) of definition 2.1 with $y=0$ shows that

$$
\rho(a x)=\rho\left(\frac{a}{b} b x\right) \leq \rho(b x) .
$$

Moreover, if $\rho$ is convex modular on $X$ and $|\alpha| \leq 1$ then, $\rho(\alpha x) \leq|\alpha| \rho(x)$ and also $\rho(x) \leq \frac{1}{2} \rho(2 x) \leq$ $\frac{\kappa}{2} \rho(x)$ if $\rho$ satisfy the $\Delta_{2}-$ condition for all $x \in X$.

Throughout this paper, $\mathbb{N}$ and $\mathbb{R}$ denote the sets of all positive integers and all real numbers, respectively.

\section{Orthogonal Stability of Eq (1.3) in Modular Spaces}

In this section we assume that the convex modular $\rho$ has the Fatou property such that satisfies the $\Delta_{2}$ - condition with $0<\kappa \leq 2$. In addition, we assume that $(E, \perp)$ denotes an orthogonality space and we define

$$
D f(x, y)=f(2 x+y)+f(2 x-y)-f(4 x)-2[f(x+y)+f(x-y)]+8 f(2 x)-10 f(x)+2 f(-x),
$$

for all $x, y \in E$ with $x \perp y$, on the other hand, we give the Hyers-Ulam-Rassias stability of the equation (1.3) in modular spaces. 
Proposition 3.1. Let $(E,\|\|$.$) with \operatorname{dim} E \geq 2$ be a real normed linear space and $X_{\rho}$ is a $\rho$-complete modular space. Let $f: E \rightarrow X_{\rho}$ be an odd mapping satisfying

$$
\rho(D f(x, y)) \leq \varepsilon\left(\|x\|^{p}+\|y\|^{p}\right),
$$

for all $x, y \in E$ with $x \perp y, \varepsilon \geq 0$ and $0<p<1$. Then there exists a unique orthogonally cubicadditive mapping $A_{c}: E \rightarrow X_{\rho}$ such that

$$
\rho\left(f(2 x)-8 f(x)-A_{c}(x)\right) \leq \frac{\varepsilon}{2-\kappa 2^{p-1}}\|x\|^{p}
$$

for all $x \in E$. Moreover

$$
A_{c}(x)=\lim _{n \rightarrow \infty} \frac{f\left(2^{n+1}\right)-8 f\left(2^{n} x\right)}{2^{n}}
$$

Proof. Letting $(x, y)=(0,0)$ in $(3.1)$, we get $f(0)=0$. Put $y=0$ in (3.1). We can do this because of (O1). Then

$$
\rho(10 f(2 x)-f(4 x)-16 f(x)) \leq \varepsilon\|x\|^{p}
$$

for all $x \in E$. Hence

$$
\rho(f(4 x)-8 f(2 x)-2(f(2 x)-8 f(x))) \leq \varepsilon\|x\|^{p}
$$

for all $x \in E$. By letting $F(x)=f(2 x)-8 f(x)$ in (3.3), we obtain

$$
\rho(F(2 x)-2 F(x)) \leq \varepsilon\|x\|^{p}
$$

for all $x \in E$. We have

$$
\rho\left(\frac{F(2 x)}{2}-F(x)\right)=\rho\left(\frac{1}{2}(F(2 x)-2 F(x))\right) \leq \frac{\varepsilon}{2}\|x\|^{p}
$$

for all $x \in E$. Replacing $x$ by $2 x$ in (3.5), we arrive to

$$
\rho\left(\frac{F\left(2^{2} x\right)}{2}-F(2 x)\right) \leq \varepsilon 2^{p-1}\|x\|^{p}
$$

for all $x \in E$. By (3.5) and (3.6), we have

$$
\begin{aligned}
\rho\left(\frac{F\left(2^{2} x\right)}{2^{2}}-F(x)\right) & =\rho\left(\frac{F\left(2^{2} x\right)}{2^{2}}-\frac{F(2 x)}{2}+\frac{F(2 x)}{2}-F(x)\right) \\
& \leq \frac{\kappa}{2} \rho\left(\frac{F(2 x)}{2}-F(x)\right)+\frac{\kappa}{2^{2}} \rho\left(\frac{F\left(2^{2} x\right)}{2}-F(2 x)\right) \\
& \leq \frac{\varepsilon}{2}\left(1+\kappa 2^{p-2}\right)\|x\|^{p}
\end{aligned}
$$

for all $x \in E$. By mathematical induction, we can easily see that

$$
\rho\left(\frac{F\left(2^{n} x\right)}{2^{n}}-F(x)\right) \leq \frac{\varepsilon}{2} \sum_{i=0}^{n-1} \kappa^{i} 2^{i(p-2)}\|x\|^{p}
$$


for all $x \in E$. Indeed, for $n=1$ the relation (3.8) is true. Assume that the relation (3.8) is true for $n$, and we show this relation rest true for $n+1$, thus we have

$$
\begin{aligned}
\rho\left(\frac{F\left(2^{n+1} x\right)}{2^{n+1}}-F(x)\right) & =\rho\left(\frac{F\left(2^{n+1} x\right)}{2^{n+1}}-\frac{F(2 x)}{2}+\frac{F(2 x)}{2}-F(x)\right) \\
& \leq \frac{\kappa}{2} \rho\left(\frac{F(2 x)}{2}-F(x)\right)+\frac{\kappa}{2^{2}} \rho\left(\frac{F\left(2^{n+1} x\right)}{2^{n}}-F(2 x)\right) \\
& \leq \frac{\kappa}{2} \frac{\varepsilon}{2}\|x\|^{p}+\frac{\varepsilon}{2} \sum_{i=0}^{n-1} \kappa^{i+1} 2^{(i+1)(p-2)}\|x\|^{p} \\
& \leq \frac{\varepsilon}{2} \sum_{i=0}^{n} \kappa^{i} 2^{i(p-2)}\|x\|^{p}
\end{aligned}
$$

hence the relation (3.8) is true for all $x \in E$ and $n \in \mathbb{N}^{*}\left(\in \mathbb{N}^{*}\right.$ : the set of positive integers). Then (3.8) become

$$
\rho\left(\frac{F\left(2^{n} x\right)}{2^{n}}-F(x)\right) \leq \frac{\varepsilon}{2} \frac{1-\left(\kappa 2^{p-2}\right)^{n}}{1-\kappa 2^{p-2}}\|x\|^{p}
$$

for all $x \in E$. Replacing $x$ by $2^{m} x$ (with $m \in \mathbb{N}^{*}$ ) in (3.9), we obtain

$$
\rho\left(\frac{F\left(2^{n+m} x\right)}{2^{n}}-F\left(2^{m} x\right)\right) \leq \frac{\varepsilon}{2} \frac{1-\left(\kappa 2^{p-2}\right)^{n}}{1-\kappa 2^{p-2}} 2^{m p}\|x\|^{p}
$$

for all $x \in E$. Whence

$$
\begin{aligned}
\rho\left(\frac{F\left(2^{n+m} x\right)}{2^{n+m}}-\frac{F\left(2^{m} x\right)}{2^{m}}\right) & \leq \frac{1}{2^{m}} \rho\left(\frac{F\left(2^{n+m} x\right)}{2^{n}}-F\left(2^{m} x\right)\right) \\
& \leq \frac{\varepsilon}{2} \frac{1-\left(\kappa 2^{p-2}\right)^{n}}{1-\kappa 2^{p-2}} 2^{m(p-1)}\|x\|^{p}
\end{aligned}
$$

for all $x \in E$. If $m, n \rightarrow \infty$ we get, the sequence $\left\{\frac{F\left(2^{n} x\right)}{2^{n}}\right\}$ is $\rho$-Cauchy sequence in the $\rho$-complete modular space $X_{\rho}$. Hence $\left\{\frac{F\left(2^{n} x\right)}{2^{n}}\right\}$ is $\rho$-convergent in $X_{\rho}$, and we well define the mapping $A_{c}=\lim _{n \rightarrow \infty} \frac{F\left(2^{n} x\right)}{2^{n}}$ from $E$ into $X_{\rho}$ satisfying

$$
\rho\left(A_{c}(x)-F(x)\right) \leq \frac{\varepsilon}{2-\kappa 2^{p-1}}\|x\|^{p},
$$

for all $x \in E$, since $\rho$ has Fatou property. To prove $A_{c}$ satisfies $D f(x, y)=0$, replace $(x, y)$ by $\left(2^{n+1} x, 2^{n+1} y\right)$ in $(3.1)$, it follows that

$$
\begin{aligned}
\rho\left(\frac{D f\left(2^{n+1} x, 2^{n+1} y\right)}{2^{n}}\right) & \leq \frac{1}{2^{n}} \rho\left(\operatorname{Df}\left(2^{n+1} x, 2^{n+1} y\right)\right) \\
& \leq \varepsilon 2^{n(p-1)+p}\left(\|x\|^{p}+\|y\|^{p}\right)
\end{aligned}
$$


for all $x, y \in E$. Again replace $(x, y)$ by $\left(2^{n} x, 2^{n} y\right)$ in $(3.1)$, it follows that

$$
\begin{aligned}
\rho\left(\frac{D f\left(2^{n} x, 2^{n} y\right)}{2^{n}}\right) & \leq \frac{1}{2^{n}} \rho\left(D f\left(2^{n+1} x, 2^{n+1} y\right)\right) \\
& \leq \varepsilon 2^{n(p-1)}\left(\|x\|^{p}+\|y\|^{p}\right)
\end{aligned}
$$

for all $x, y \in E$. By (3.13) and (3.14), we get

$$
\begin{aligned}
\rho\left(\frac{D f\left(2^{n+1} x, 2^{n+1} y\right)-8 D f\left(2^{n} x, 2^{n} y\right)}{2^{n}}\right) & \leq \frac{\kappa}{2} \rho\left(\frac{D f\left(2^{n+1} x, 2^{n+1} y\right)}{2^{n}}\right)+\frac{\kappa^{4}}{2} \rho\left(\frac{D f\left(2^{n} x, 2^{n} y\right)}{2^{n}}\right) \\
& \leq \frac{\kappa}{2} \varepsilon 2^{n(p-1)+p}\left(\|x\|^{p}+\|y\|^{p}\right)+\frac{\kappa^{4}}{2} \varepsilon 2^{n(p-1)}\left(\|x\|^{p}+\|y\|^{p}\right)
\end{aligned}
$$

If $n \rightarrow \infty$ then, we conclude that $D A_{c}(x, y)=0$, for all $x, y \in E$ with $x \perp y$. Therefore $A_{c}: E \rightarrow$ $X_{\rho}$ is an orthogonally cubic-additive mapping satisfying (1.3). To prove the uniqueness, assume $A_{c}^{\prime}: E \rightarrow X_{\rho}$ to be another orthogonally cubic-additive mapping satisfying (3.12). Then, for each $x, y \in E$ and for all $m \in \mathbb{N}$ on has

$$
\begin{aligned}
\rho\left(A_{c}(x)-A_{c}^{\prime}(x)\right) & =\rho\left(\frac{A_{c}\left(2^{m} x\right)}{2^{m}}-\frac{A_{c}^{\prime}\left(2^{m} x\right)}{2^{m}}\right) \\
& \leq \frac{\kappa}{2^{m+1}}\left[\rho\left(A_{c}\left(2^{m} x\right)-F\left(2^{m} x\right)\right)+\rho\left(A_{c}^{\prime}\left(2^{m} x\right)-F\left(2^{m} x\right)\right)\right] \\
& \leq \frac{\kappa \varepsilon 2^{m(p-1)}}{2-\kappa 2^{p-1}}\|x\|^{p}
\end{aligned}
$$

If $m \rightarrow \infty$, we obtain $A_{c}=A_{c}^{\prime}$.

Q.E.D.

Proposition 3.2. Let $(E,\|\|$.$) with \operatorname{dim} E \geq 2$ be a real normed linear space and $X_{\rho}$ is a $\rho$-complete modular space. Let $f: E \rightarrow X_{\rho}$ be an odd mapping satisfying

$$
\rho(D f(x, y)) \leq \varepsilon\left(\|x\|^{p}+\|y\|^{p}\right),
$$

for all $x, y \in E$ with $x \perp y, \varepsilon \geq 0$ and $0<p<3$. Then there exists a unique orthogonally cubicadditive mapping $C_{a}: E \rightarrow X_{\rho}$ such that

$$
\rho\left(f(2 x)-2 f(x)-C_{a}(x)\right) \leq \frac{\varepsilon}{8-\kappa 2^{p-1}}\|x\|^{p}
$$

for all $x \in E$. Moreover

$$
C_{a}(x)=\lim _{n \rightarrow \infty} \frac{f\left(2^{n+1}\right)-2 f\left(2^{n} x\right)}{8^{n}}
$$

Proof. By (3.3), we have

$$
\rho(f(4 x)-2 f(2 x)-8(f(2 x)-2 f(x))) \leq \varepsilon\|x\|^{p}
$$

for all $x \in E$. By letting $G(x)=f(2 x)-2 f(x)$ in (3.17), we get

$$
\rho\left(\frac{G(2 x)}{8}-G(x)\right) \leq \frac{\varepsilon}{8}\|x\|^{p}
$$


for all $x \in E$. Now replacing $x$ by $2 x$ in (3.18), we find

$$
\rho\left(\frac{G\left(2^{2} x\right)}{8}-G(2 x)\right) \leq \frac{\varepsilon 2^{p}}{8}\|x\|^{p}
$$

for all $x \in E$. Then

$$
\rho\left(\frac{G\left(2^{2} x\right)}{8^{2}}-\frac{G(2 x)}{8}\right) \leq \frac{\varepsilon 2^{p-3}}{8}\|x\|^{p}
$$

for all $x \in E$. From (3.18) and (3.20), we have

$$
\begin{aligned}
\rho\left(\frac{G\left(2^{2} x\right)}{8^{2}}-G(x)\right) & \leq \frac{\kappa}{2} \rho\left(\frac{G\left(2^{2} x\right)}{8^{2}}-\frac{G(2 x)}{8}\right)+\frac{\kappa}{2} \rho\left(\frac{G(2 x)}{8}-G(2 x)\right) \\
& \leq \frac{\varepsilon}{8}\left(1+\frac{\kappa}{2} 2^{p-3}\right)\|x\|^{p}
\end{aligned}
$$

for all $x \in E$. In general, using induction on a positive integer $n$, we obtain

$$
\begin{aligned}
\rho\left(\frac{G\left(2^{n} x\right)}{8^{n}}-G(x)\right) & \leq \frac{\varepsilon}{8} \sum_{i=0}^{n-1}\left(\frac{\kappa}{2}\right)^{i} 2^{i(p-3)}\|x\|^{p} \\
& =\frac{\varepsilon}{8} \frac{1-\left(\kappa 2^{p-4}\right)^{n}}{1-\kappa 2^{p-4}}\|x\|^{p}
\end{aligned}
$$

for all $x \in E$. Replacing $x$ by $2^{m} x$ (with $m \in \mathbb{N}^{*}$ ) in (3.21), we get

$$
\rho\left(\frac{G\left(2^{n+m} x\right)}{8^{n}}-G\left(2^{m} x\right)\right) \leq \frac{\varepsilon}{8} \frac{1-\left(\kappa 2^{p-4}\right)^{n}}{1-\kappa 2^{p-4}} 2^{m p}\|x\|^{p}
$$

for all $x \in E$. Whence

$$
\rho\left(\frac{G\left(2^{n+m} x\right)}{8^{n+m}}-\frac{F\left(2^{m} x\right)}{8^{m}}\right) \leq \frac{\varepsilon}{8} \frac{1-\left(\kappa 2^{p-4}\right)^{n}}{1-\kappa 2^{p-4}} 2^{m(p-3)}\|x\|^{p}
$$

for all $x \in E$. If $m, n \rightarrow \infty$ we get, the sequence $\left\{\frac{G\left(2^{n} x\right)}{8^{n}}\right\}$ is $\rho$-Cauchy sequence in the $\rho$-complete modular space $X_{\rho}$. Hence $\left\{\frac{G\left(2^{n} x\right)}{8^{n}}\right\}$ is $\rho$-convergent in $X_{\rho}$, and we well define the mapping $C_{a}=\lim _{n \rightarrow \infty} \frac{G\left(2^{n} x\right)}{8^{n}}$ from $E$ into $X_{\rho}$ satisfying

$$
\rho\left(C_{a}(x)-G(x)\right) \leq \frac{\varepsilon}{8-\kappa 2^{p-1}}\|x\|^{p},
$$

for all $x \in E$, since $\rho$ has Fatou property. The rest of the proof is similar to the proof of proposition 3.1 .

Q.E.D.

Theorem 3.3. Let $(E,\|\|$.$) with \operatorname{dim} E \geq 2$ be a real normed linear space and $X_{\rho}$ is a $\rho$-complete modular space. Let $f: E \rightarrow X_{\rho}$ be an odd mapping satisfying

$$
\rho(D f(x, y)) \leq \varepsilon\left(\|x\|^{p}+\|y\|^{p}\right),
$$


for all $x, y \in E$ with $x \perp y, \varepsilon \geq 0$ and $0<p<1$. Then there exists a unique orthogonally cubicadditive mapping $A C: E \rightarrow X_{\rho}$ such that

$$
\rho(f(x)-A C(x)) \leq \frac{\kappa \varepsilon}{12}\left\{\frac{1}{2-\kappa 2^{p-1}}+\frac{1}{8-\kappa 2^{p-1}}\right\}\|x\|^{p}
$$

for all $x \in E$. Moreover

$$
A C(x)=\frac{-1}{6} A_{c}(x)+\frac{1}{6} C_{a}(x)
$$

for all $x \in E$.

Proof. By proposition 3.1 and proposition 3.2, we have

$$
\begin{aligned}
\rho(f(x)-A C(x)) & =\rho\left(f(x)+\frac{1}{6} A_{c}(x)-\frac{1}{6} C_{a}(x)\right) \\
& =\rho\left(\frac{-1}{6}\left[f(2 x)-8 f(x)-A_{c}(x)\right]+\frac{1}{6}\left[f(2 x)-2 f(x)-C_{a}(x)\right]\right) \\
& \leq \frac{\kappa}{12}\left\{\rho\left(\left[f(2 x)-8 f(x)-A_{c}(x)\right]\right)+\rho\left(\left[f(2 x)-2 f(x)-C_{a}(x)\right]\right)\right\} \\
& \leq \frac{\kappa \varepsilon}{12}\left\{\frac{1}{2-\kappa 2^{p-1}}+\frac{1}{8-\kappa 2^{p-1}}\right\}\|x\|^{p}
\end{aligned}
$$

for all $x \in E$.

Q.E.D.

Remark 3.4. [16] Let $f: E \rightarrow X_{\rho}$ be an even mapping satisfying (1.3) (with $x \perp y$ ), then $f=0$ on $E$.

Proposition 3.5. Let $(E,\|\cdot\|)$ with $\operatorname{dim} E \geq 2$ be a real normed linear space and $X_{\rho}$ is a $\rho$-complete modular space. Let $f: E \rightarrow X_{\rho}$ be an even mapping satisfying

$$
\rho(D f(x, y)) \leq \varepsilon\left(\|x\|^{p}+\|y\|^{p}\right),
$$

for all $x, y \in E$ with $x \perp y, \varepsilon \geq 0$ and $0<p<1$. Then

$$
\rho(f(x)) \leq \frac{\varepsilon}{2} \frac{1+\kappa 2^{p-2}}{1-\kappa 2^{p-2}}\|x\|^{p}
$$

for all $x \in E$.

Proof. Letting $(x, y)=(0,0)$ in $(3.27)$, we get $f(0)=0$. Putting $(x, y)=(0, x)$ in $(3.27)$, we obtain

$$
\rho(f(x))=\rho\left(\frac{1}{2} 2 f(x)\right) \leq \frac{1}{2} \rho(2 f(x)) \leq \frac{\varepsilon}{2}\|x\|^{p}
$$

for all $x \in E$. Replacing $x$ by $2 x$ in (3.29), we find

$$
\rho(f(2 x)) \leq \frac{\varepsilon 2^{p}}{2}\|x\|^{p}
$$


for all $x \in E$. Thus

$$
\begin{aligned}
\rho\left(\frac{1}{2} f(2 x)-f(x)\right) & \leq \frac{\kappa}{2^{2}} \rho(f(2 x))+\frac{\kappa}{2} \rho(f(x)) \\
& \leq \frac{\varepsilon}{2}\left(1+\kappa 2^{p-2}\right)\|x\|^{p}
\end{aligned}
$$

for all $x \in E$. Now replacing $x$ by $2 x$ in (3.31), we get

$$
\begin{aligned}
\rho\left(\frac{f\left(2^{2} x\right)}{2^{2}}-\frac{f(2 x)}{2}\right) & \leq \frac{1}{2} \rho\left(\frac{1}{2} f\left(2^{2} x\right)-f(2 x)\right) \\
& \leq \frac{\varepsilon}{2}\left(1+\kappa 2^{p-2}\right) 2^{p-1}\|x\|^{p}
\end{aligned}
$$

for all $x \in E$. It follows that

$$
\begin{aligned}
\rho\left(\frac{f\left(2^{2} x\right)}{2^{2}}-f(x)\right) & =\rho\left(\frac{f\left(2^{2} x\right)}{2^{2}}-\frac{f(2 x)}{2}+\frac{f(2 x)}{2}-f(x)\right) \\
& \leq \frac{\kappa}{2} \rho\left(\frac{f\left(2^{2} x\right)}{2^{2}}-\frac{f(2 x)}{2}\right)+\frac{\kappa}{2} \rho\left(\frac{f(2 x)}{2}-f(x)\right) \\
& \leq \frac{\varepsilon\left(1+\kappa 2^{p-2}\right)}{2}\left(1+\frac{\kappa}{2} 2^{p-1}\right)\|x\|^{p}
\end{aligned}
$$

for all $x \in E$. In general, using induction on a positive integer $n$, we obtain

$$
\begin{aligned}
\rho\left(\frac{f\left(2^{n} x\right)}{2^{n}}-f(x)\right) & \leq \frac{\varepsilon\left(1+\kappa 2^{p-2}\right)}{2} \sum_{i=0}^{n-1}\left(\frac{\kappa}{2}\right)^{i} 2^{i(p-1)}\|x\|^{p} \\
& =\frac{\varepsilon\left(1+\kappa 2^{p-2}\right)}{2} \frac{1-\left(\kappa 2^{p-2}\right)^{n}}{1-\kappa 2^{p-2}}\|x\|^{p}
\end{aligned}
$$

for all $x \in E$. Since $\left\{\frac{f\left(2^{n} x\right)}{2^{n}}\right\}$ is $\rho$-Cauchy sequence in the $\rho$-complete modular space $X_{\rho}$ (the proof is similar to that of proposition 3.1). Hence $\left\{\frac{f\left(2^{n} x\right)}{2^{n}}\right\}$ is $\rho$-convergent in $X_{\rho}$, and we well define the mapping $A_{e}=\lim _{n \rightarrow \infty} \frac{f\left(2^{n} x\right)}{2^{n}}$ from $E$ into $X_{\rho}$ satisfying

$$
\rho\left(f(x)-A_{e}(x)\right) \leq \frac{\varepsilon}{8-\kappa 2^{p-1}}\|x\|^{p},
$$

for all $x \in E$, since $\rho$ has Fatou property. The proof of $D A_{e}(x, y)=0$ (with $x \perp y$ ) is similar to the proof of proposition 3.1. $A_{e}$ is even orthogonally cubic-additive mapping, by remark $3.4, A_{e}(x)=0$ for all $x \in E$, and this completes the proof.

Q.E.D.

Theorem 3.6. Let $(E,\|\|$.$) with \operatorname{dim} E \geq 2$ be a real normed linear space and $X_{\rho}$ is a $\rho$-complete modular space. Let $f: E \rightarrow X_{\rho}$ be a mapping satisfying

$$
\rho(D f(x, y)) \leq \varepsilon\left(\|x\|^{p}+\|y\|^{p}\right),
$$


for all $x, y \in E$ with $x \perp y, \varepsilon \geq 0$ and $0<p<1$. Then there exists a unique orthogonally cubicadditive mapping $A C: E \rightarrow X_{\rho}$ such that

$$
\rho(f(x)-A C(x)) \leq \frac{\kappa \varepsilon}{4}\left\{\frac{1+\kappa 2^{p-2}}{1-\kappa 2^{p-2}}+\frac{\kappa}{6}\left[\frac{1}{2-\kappa 2^{p-1}}+\frac{1}{8-\kappa 2^{p-1}}\right]\right\}
$$

for all $x \in E$.

Proof. Let $f^{e}$ and $f^{0}$ are even and odd part of $f$ such that $f^{e}(x)=\frac{f(x)+f(-x)}{2}, f^{o}(x)=\frac{f(x)-f(-x)}{2}$. Then we have

$$
\begin{aligned}
\rho\left(D f^{e}(x, y)\right) & =\rho\left(\frac{D f(x, y)+D f(-x,-y)}{2}\right) \leq \frac{1}{2} \rho(D f(x, y))+\frac{1}{2} \rho(D f(-x,-y)) \\
& \leq \varepsilon\left(\|x\|^{p}+\|y\|^{p}\right) .
\end{aligned}
$$

By proposition 3.5, we have

$$
\rho\left(f^{e}(x)\right) \leq \frac{\varepsilon}{2} \frac{1+\kappa 2^{p-2}}{1-\kappa 2^{p-2}}\|x\|^{p}
$$

for all $x \in E$. Similarly we obtain

$$
\begin{aligned}
\rho\left(D f^{o}(x, y)\right) & =\rho\left(\frac{D f(x, y)-D f(-x,-y)}{2}\right) \leq \frac{1}{2} \rho(D f(x, y))+\frac{1}{2} \rho(D f(-x,-y)) \\
& \leq \varepsilon\left(\|x\|^{p}+\|y\|^{p}\right) .
\end{aligned}
$$

By theorem 3.3, there exists a unique orthogonally cubic-additive mapping $A C: E \rightarrow X_{\rho}$ such that

$$
\rho\left(f^{o}(x)-A C(x)\right) \leq \frac{\kappa \varepsilon}{12}\left\{\frac{1}{2-\kappa 2^{p-1}}+\frac{1}{8-\kappa 2^{p-1}}\right\}\|x\|^{p}
$$

for all $x \in E$. It follows from (3.38) and (3.39) that

$$
\begin{aligned}
\rho(f(x)-A C(x)) & =\rho\left(f^{e}(x)+f^{o}(x)-A C(x)\right) \leq \frac{\kappa}{2} \rho\left(f^{e}(x)\right)+\frac{\kappa}{2} \rho\left(f^{o}(x)-A C(x)\right) \\
& \leq \frac{\kappa \varepsilon}{4} \frac{1+\kappa 2^{p-2}}{1-\kappa 2^{p-2}}\|x\|^{p}+\frac{\kappa}{24}\left\{\frac{1}{2-\kappa 2^{p-1}}+\frac{1}{8-\kappa 2^{p-1}}\right\}\|x\|^{p} \\
& =\frac{\kappa \varepsilon}{4}\left\{\frac{1+\kappa 2^{p-2}}{1-\kappa 2^{p-2}}+\frac{\kappa}{6}\left[\frac{1}{2-\kappa 2^{p-1}}+\frac{1}{8-\kappa 2^{p-1}}\right]\right\}
\end{aligned}
$$

for all $x \in E$.

Q.E.D.

Corollary 3.6.1. [16] Let $(E,\|\cdot\|)$ with $\operatorname{dim} E \geq 2$ be a real normed linear space and $X$ is a Banach space. Let $f: E \rightarrow X$ be mappings satisfying

$$
\|D f(x, y)\| \leq \varepsilon\left(\|x\|^{p}+\|y\|^{p}\right),
$$

for all $x, y \in E$ with $x \perp y, \varepsilon \geq 0$ and $0<p<1$. Then there exists a unique orthogonally cubicadditive mapping $A C: E \rightarrow X$ such that

$$
\|f(x)-A C(x)\| \leq \frac{\varepsilon}{2}\left\{\frac{1+2^{p-1}}{1-2^{p-1}}+\frac{1}{3}\left[\frac{1}{2-2^{p}}+\frac{1}{8-2^{p}}\right]\right\}
$$

for all $x \in E$. 
Proof. It is well known that every normed space is a modular space with the modular $\rho(x)=\|x\|$ and $\kappa=2$.

A convex function $\varphi$ defined on the interval $[0, \infty)$, non-decreasing and continuous for $\alpha \geq 0$ and such that $\varphi(0)=0, \varphi(\alpha)>0$ for $\alpha>0, \varphi(\alpha) \rightarrow \infty$ as $\alpha \rightarrow \infty$, is called an Orlicz function. The Orlicz function $\varphi$ satisfies the $\Delta_{2}$-condition if there exist $k>0$ such that $\varphi(2 \alpha) \leq k \varphi(\alpha)$ for all $\alpha>0$. Let $(\Omega, \Sigma, \mu)$ be a measure space. Let us consider the space $L_{\mu}^{0}$ consisting of all measurable real-valued (or complex-valued) function on $\Omega$. Define for every $f \in L_{\mu}^{0}$ the Orlicz modular $\rho_{\varphi}(f)$ by the formula

$$
\rho_{\varphi}(f)=\int_{\Omega} \varphi(|f|) d \mu
$$

The associated modular function space with respect to this modular is called an Orlicz space, and will be denoted by $L_{\mu}^{\varphi}(\Omega)$ or briefly $L^{\varphi}$. In other words

$$
L^{\varphi}=\left\{f \in L_{\mu}^{0}: \rho_{\varphi}(\lambda f) \rightarrow 0 \text { as } \lambda \rightarrow 0\right\}
$$

or equivalently as

$$
L^{\varphi}=\left\{f \in L_{\mu}^{0}: \rho_{\varphi}(\lambda f)<\infty \text { for some } \lambda>0\right\} .
$$

It is known that the Orlicz space $L^{\varphi}$ is $\rho_{\varphi}$-complete. Moreover, $\left(L^{\varphi},\|\cdot\|_{\rho_{\varphi}}\right)$ is a Banach space, where the Luxemburg norm $\|\cdot\|_{\rho_{\varphi}}$ is defined as follows

$$
\|f\|_{\rho_{\varphi}}=\inf \left\{\lambda>0: \int_{\Omega} \varphi\left(\frac{|f|}{\lambda}\right) d \mu \leq 1\right\}
$$

Moreover, if $\ell$ is the space of sequences $x=\left(x_{i}\right)_{i=1}^{\infty}$ with real or complex terms $x_{i}, \varphi=\left(\varphi_{i}\right)_{i=1}^{\infty}, \varphi_{i}$ are Orlicz functions and $\pi_{\varphi}(x)=\Sigma_{i=1}^{\infty} \varphi_{i}\left(\left|x_{i}\right|\right)$, we shall write $\ell^{\varphi}$ in place of $L^{\varphi}$. The space $\ell^{\varphi}$ is called the generalized Orlicz sequence space. The motivation for the study of modular spaces (and Orlicz spaces) and many examples are detailed in $[14,15]$. Now, we give a following examples.

Example 3.7. Let $(E,\|\|$.$) with \operatorname{dim} E \geq 2$ be a real normed linear space, $\varphi$ is an Orlicz function and satisfy the $\Delta_{2}$-condition with $0<\kappa \leq 2$. Let $f: E \rightarrow L^{\varphi}$ be a mapping satisfying

$$
\int_{\Omega} \varphi(|D f(x, y)|) d \mu \leq \varepsilon\left(\|x\|^{p}+\|y\|^{p}\right),
$$

for all $x, y \in E$ with $x \perp y, \varepsilon \geq 0$ and $0 \leq p<1$. Then there exists a unique orthogonally cubicadditive mapping $A C: E \rightarrow L^{\varphi}$ such that

$$
\int_{\Omega} \varphi(|f(x)-A C(x)|) d \mu \leq \frac{\kappa \varepsilon}{4}\left\{\frac{1+\kappa 2^{p-2}}{1-\kappa 2^{p-2}}+\frac{\kappa}{6}\left[\frac{1}{2-\kappa 2^{p-1}}+\frac{1}{8-\kappa 2^{p-1}}\right]\right\}
$$

for all $x \in E$.

Example 3.8. Let $(E,\|\|$.$) with \operatorname{dim} E \geq 2$ be a real normed linear space, $\widehat{\varphi}=\left(\varphi_{i}\right)$ be sequence of Orlicz functions satisfying the $\Delta_{2}$-condition with $0<\kappa \leq 2$ and let $\left(\ell^{\widehat{\varphi}}, \pi_{\hat{\varphi}}\right)$ be generalized Orlicz sequence space associated to $\widehat{\varphi}=\left(\varphi_{i}\right)$. Let $f: E \rightarrow \ell^{\widehat{\varphi}}$ be a mapping satisfying

$$
\pi_{\widehat{\varphi}}(D f(x, y)) \leq \varepsilon\left(\|x\|^{p}+\|y\|^{p}\right),
$$


for all $x, y \in E$ with $x \perp y, \varepsilon \geq 0$ and $0 \leq p<1$. Then there exists a unique orthogonally cubicadditive mapping $A C: E \rightarrow \ell_{\widehat{\varphi}}$ such that

$$
\pi_{\widehat{\varphi}}(f(x)-A C(x)) \leq \frac{\kappa \varepsilon}{4}\left\{\frac{1+\kappa 2^{p-2}}{1-\kappa 2^{p-2}}+\frac{\kappa}{6}\left[\frac{1}{2-\kappa 2^{p-1}}+\frac{1}{8-\kappa 2^{p-1}}\right]\right\}
$$

for all $x \in E$.

\section{References}

[1] M. Adam and S. Czerwik, On the stability of the quadratic functional equation in topological spaces, Banach Journal of mathematical Analysis, 1 (2007) no.2, 245-251.

[2] T. Aoki, On the stability of the linear transformation in Banach spaces, J. Math. Soc., Japan, 2 (1950), 64-66.

[3] P. W. Cholewa, Remarks on the stability of functional equations, Aequationes Math. 27 (1984), 7686.

[4] S. Czerwik, On the stability of the quadratic mapping in normed spaces, Abh. Math. Sem. Univ. Hamburg 62 (1992), 5964.

[5] Iz. El-Fassi and S. Kabbaj, Hyers-Ulam-Rassias Stability of Orthogonal Quadratic Functional Equation in Modular Spaces, General Mathematics Notes (GMN), vol. 26, No. 1 (2015), 61-73.

[6] M. Eshaghi Gordji and M. Bavand Savadkoumi, Approximation of generalized homomorphisms in quasi-Banach algebra, Aalele Univ. Ovidius Constata, Math. Series 17, (2009), 203-213.

[7] P. Găvruta, A generalization of Hyers-Ulam-Rassias Stability of the approximately additive mappings, J. Math. Anal. App. 184 (1994), 431-436.

[8] R. Ger and J. Sikorska Stability of the orthogonal additivity, Bull. Polish Acad. Sci. Math, 43 (1995), 143-151.

[9] S. Gudder and D. Strawther, Orthogonally additive and orthogonally increasing function on vector spaces, Pacific J. Math., 58 (1995), 427-436.

[10] D. H. Hyers, On the stability of the linear functional equation, Proc. Nat. Acad. Sci., U.S.A., 27 (1941), 222-224.

[11] S.-M. Jung, On the HyersUlam stability of the functional equations that have the quadratic property, J. Math. Anal. Appl. 222 (1998), 126137.

[12] M. Krbec, Modular interpolation spaces, Z. Anal. Anwendungen 1 (1982), 25-40.

[13] A. Najati and M. B. Moghimi, Stability of a functional equation deriving from quadratic and additive functions in quasi-Banach spaces, J. Math. Anal, 337 (2008), 399-415. 
[14] H. Nakano, Modulared Semi-Ordered Linear Spaces, in: Tokyo Math. Book Ser., Vol. 1, Maruzen Co., Tokyo, 1950.

[15] W. Orlicz, Collected Papers, Vols. I, II, PWN, Warszawa, 1988.

[16] S. Ostadbashi and J. Kazemzadeh, Orthogonal stability of mixed type additive and cubic functional equations, Int. J. Nonlinear Anal. Appl. 6 (2015) No. 1, 35-43

[17] Th. M. Rassias, On the stability of the linear mapping in Banach spaces, Proc. Amer. Math. Soc., 72 (1978), 297-300.

[18] J. Rätz, On orthogonally additive mappings, Aequations Math., 28 (1985), 35-49.

[19] Gh. Sadeghi, A fixed point approach to stability of functional equations in modular spaces, Bull. Malays. Math. Sci. Soc. 37 (2) (2014), 333-344.

[20] F. Skof, Proprit locali e approssimazione di operatori, Rend. Sem. Mat. Fis. Milano 53 (1983), 113129.

[21] S. M. Ulam, Problems in Modern Mathematics, Chapter IV, Science Editions, Wiley, New York, 1960. 